\title{
FURTHER GENERALISATIONS OF THE KUMMER-SCHWARZ EQUATION: ALGEBRAIC AND SINGULARITY PROPERTIES
}

\author{
R. Sinuvasan ${ }^{a, c, *}$, K. Krishnakumar ${ }^{a}$, K. M. TAmizhmani ${ }^{a}$, \\ P. G. L. LEACH ${ }^{b}$ \\ ${ }^{a}$ Department of Mathematics, Pondicherry University, Kalapet, India 605014 \\ ${ }^{b}$ School of Mathematics, Statistics and Computer Science, University of KwaZulu-Natal, Private Bag X54001, \\ Durban 4000, Republic of South Africa and Institute for Systems Science, Department of Mathematics, Durban \\ University of Technology, POB 1334, Durban 4000, Republic of South Africa \\ ${ }^{c}$ Department of Mathematics, SASTRA Deemed University, Thanjavur, India 613401 \\ * corresponding author: rsinuvasan@gmail.com
}

Abstract. The Kummer-Schwarz Equation, $2 y^{\prime} y^{\prime \prime \prime}-3\left(y^{\prime \prime}\right)^{2}=0$, has a generalisation, $(n-$ 1) $y^{(n-2)} y^{(n)}-n y^{(n-1)^{2}}=0$, which shares many properties with the parent form (see Sinuvasan R, Tamizhmani K M \& Leach P G L, Algebraic and singularity properties of a class of generalisations of the Kummer-Schwarz equation differ, Equ Dyn Syst (2016), doi:10.1007/s12591-016-0327-5) in terms of symmetry and singularity. All equations of the class are integrable in closed form. Here we introduce a new class, $(n+q-2) y^{(n-2)} y^{(n)}-(n+q-1) y^{(n-1)^{2}}=0$, which has different integrability and singularity properties.

KEYWORDS: Kummer-Schwarz; symmetries; singularities; integrability.

\section{INTRODUCTION}

In 12] we delineated the properties of the class of equations

$$
(n-1) y^{(n-2)} y^{(n)}-n\left(y^{(n-1)}\right)^{2}=0, \quad n=2,3, \ldots
$$

in terms of symmetry, singularity and integrability. The class (1) differed from the class with general numerical coefficients in that the number of Lie point symmetries was one greater for general values of $n$ (with $n=2$ being an exceptional case). In the case of the Kummer-Schwarz Equation [4] it was two greater, being the six comprising the direct sum of two $\operatorname{sl}(2, R)$ subalgebras.

Here we consider the generalisation

$$
\begin{array}{r}
(n+q-2) y^{(n-2)} y^{(n)}-(n+q-1) y^{(n-1)^{2}}=0, \\
n=2,3, \ldots,
\end{array}
$$

where $q$ is a real number, subsequently to be more precisely defined.

We examine 2 in terms of its Singularity Properties, Symmetry Properties and general Integrability. We commence with the singularity properties of (2) and see that a successful satisfaction of the requirements imposes a constraint on the permissible values of $q$ for the solution to be analytic. We turn to the symmetry properties of (2) and find some unexpected results. In terms of integrability there are no restrictions upon the value of $q$ in a formal sense although in practice there may be restrictions.

\section{Singularity Analysis}

We examine the sequence of equations introduced above in terms of singularity analysis. We follow the general method as outlined in [10, 14] with the modification for negative nongeneric resonances introduced by Andriopoulos et al. 1 .

Theorem. The exponent of the leading-order term and the resonances of the $n$th member of the sequence of equations,

$$
\begin{gathered}
(n+q-2) y^{(n-2)} y^{(n)}-(n+q-1)\left(y^{(n-1)}\right)^{2}=0, \\
n \in N, n>1,
\end{gathered}
$$

are $p=-q$ and $s=-1,0, q, 1+q, \ldots, n-3+q$.

Proof. We substitute $y=\alpha \chi^{p}$, where $\chi=x-x_{0}$ and $x_{0}$ is the location of the putative singularity. We remove a common factor $p^{2}(p-1)^{2} \cdots(p-n+3)^{2}(p-$ $n+2)$. The values of $p$ removed are all positive and so of no relevance to the singularity analysis. The remaining terms are

$$
(n+q-2)(p-n+1)-(n+q-1)(p-n+2)
$$

which, when put equal to zero, give the singularity to be $p=-q$.

We write $y=\alpha \chi^{-q}+\mu \chi^{-q+s}$ and substitute into (3). We remove the common factors $\chi^{-2}$ and obtain

$$
\begin{gathered}
(-q)(-q-1) \cdots(-q-n+3)(-q+s)(-q+s-1) \cdots \\
\cdots(-q+s-n+3)
\end{gathered}
$$

This immediately gives the resonances, $s=q, q+1$, $\ldots, q+n-3$, which are all positive. The remaining terms are

$$
\begin{aligned}
& (n+q-2)(-q-n+2)(-q-n+1) \\
& +(n+q-2)(-q+s-n+2)(-q+s-n+1) \\
& \quad-2(n+q-1)(-q-n+2)(-q+s-n+2) .
\end{aligned}
$$


When this equated to zero, we obtain the two additional resonances $s=-1,0$.

Apart from the generic resonance of -1 all of the resonances are nonnegative numbers which contain the positive number $q$. When the resulting expansion make sense, the Laurent Expansion is a Right Painlevé Series [2].

We illustrate the method with the fifth-order equation,

$$
(q+3) y^{\prime \prime \prime} y^{(5)}-(q+4)\left(y^{(4)}\right)^{2}=0 .
$$

To determine the leading-order behaviour we set $y=\alpha \chi^{p}$, where $\chi=x-x_{0}$ and $x_{0}$ is the location of the putative singularity. We obtain

$$
\begin{aligned}
& (q+3) \alpha^{2} p^{2}(p-1)^{2}(p-2)^{2}(p-3)(p-4) \chi^{2 p-8} \\
& -(q+4) \alpha^{2} p^{2}(p-1)^{2}(p-2)^{2}(p-3)^{2} \chi^{2 p-8}
\end{aligned}
$$

which is zero if $(q+3)(p-4)=(q+4)(p-3)$, i.e., $p=-q$. Note that the coefficient of the leading-order term is arbitrary.

To establish the terms at which the remaining constants of integration occur in the Laurent Expansion we make the substitution

$$
y=\alpha \chi^{-q}+m \chi^{-q+s} .
$$

The various values $s$ may take are determined by those values of $s$ which make the coefficient of $m$ so that $m$ is arbitrary. The coefficient of $m$ is a fifth-order polynomial the roots of which are

$$
s=-1,0, q, 1+q, 2+q
$$

\section{Solution OF the General EQUATION}

Equation (2), up to a multiplicative constant, may be written as

$$
\frac{\mathrm{d}^{2} y^{(n-2)}(x)^{-\frac{1}{n+q-2}}}{\mathrm{~d} x^{2}}=0
$$

which is readily integrated to give the general solution of (2) as

$$
y=(-1)^{n} \frac{(a x+b)^{-q}}{a^{n-2}(q+n-3)^{(n-3)}}+\sum_{i=0}^{n-3} c_{i} x^{i},
$$

where the notation $(q+n-3)^{(n-3)}$ denotes the rising Pochhammer Symbol and means $q(q+1) \cdots(q+n-3)$.

We note that the solution of (2) exists no matter the value of $q$. Naturally the utility of the solution depends upon the value of $q$ because we are dealing with values in the complex plane.

\section{Symmetry Properties}

The symmetry properties of (2) are rather complex and to give an indication of the complexity we quote the results for a small sample of equations. We commence with $n=2$ for which we obtain the symmetries,

$$
\begin{array}{r}
\left\{\frac{\partial}{\partial x}, y \frac{\partial}{\partial y}, x \frac{\partial}{\partial x}, y^{\frac{1}{q}+1} \frac{\partial}{\partial y}, x y^{\frac{1}{q}+1} \frac{\partial}{\partial y}, q y^{-1 / q} \frac{\partial}{\partial x}\right. \\
\left.x y \frac{\partial}{\partial y}-\frac{x^{2}}{q} \frac{\partial}{\partial x}, y^{1-\frac{1}{q}} \frac{\partial}{\partial y}-\frac{1}{q}\left(x y^{-1 / q}\right) \frac{\partial}{\partial x}\right\}
\end{array}
$$

with the algebra $\operatorname{sl}(3, R)$. The transformation to the archetypal second-order equation (up to a multiplicative factor) is $y(x) \rightarrow w(x)^{-q}$. The transformation follows from inspection of the structures of the symmetries above.

In the case of the third-order equation, i.e., $n=3$, we obtain four possible algebraic structures. These are

$$
\begin{gathered}
\left\{\frac{\partial}{\partial y}, \frac{\partial}{\partial x}, y \frac{\partial}{\partial y}, x \frac{\partial}{\partial x}\right\}, \\
\left\{\frac{\partial}{\partial y}, \frac{\partial}{\partial x}, y \frac{\partial}{\partial y}, x \frac{\partial}{\partial x}, y^{2} \frac{\partial}{\partial y}, x^{2} \frac{\partial}{\partial x}\right\}, \\
\left\{\frac{\partial}{\partial y}, \frac{\partial}{\partial x}, y \frac{\partial}{\partial y}, x \frac{\partial}{\partial y}, x \frac{\partial}{\partial x}, x^{2} \frac{\partial}{\partial y}, x^{2} \frac{\partial}{\partial x}+2 x y \frac{\partial}{\partial y}\right\}, \\
\left\{\frac{\partial}{\partial y}, \frac{\partial}{\partial x}, y \frac{\partial}{\partial y}, y \frac{\partial}{\partial x}, x \frac{\partial}{\partial x}, y^{2} \frac{\partial}{\partial x}, 2 x y \frac{\partial}{\partial x}+y^{2} \frac{\partial}{\partial y}\right\}
\end{gathered}
$$

corresponding to general $q, q=1, q=-2$ and $q=-\frac{1}{2}$. Clearly the latter two cases did not come within the purview of the singularity analysis discussed above. For general $q$ the algebra is $A_{2} \oplus A_{2}$ which can also be written as $2 A_{2}$. (We make use of the Mubarakzyanov Classification Scheme [5] 8] (see also [9, 11, 13]) throughout this paper.) In the case of $q=1$ we have the well-known Kummer-Schwarz Equation with the algebra $2 s l(2, R)$ or $2 A_{3,8}$. For the values of $q-2$ and $-\frac{1}{2}$ the algebra is the same and is the maximal algebra for a third-order equation. For $q=-2$ the representation is the standard representation for $y^{\prime \prime \prime}=0$. The representation for $q=-\frac{1}{2}$ has not been reported before. However, the latter result follows from an interchange of $x$ and $y$.

The fourth-order equation is the prototype for subsequent equations and we find the symmetries

$$
\begin{gathered}
\left\{\frac{\partial}{\partial y}, x \frac{\partial}{\partial y}, y \frac{\partial}{\partial y}, \frac{\partial}{\partial x}, x \frac{\partial}{\partial x}\right\} \\
\left\{\frac{\partial}{\partial y}, x \frac{\partial}{\partial y}, y \frac{\partial}{\partial y}, \frac{\partial}{\partial x}, x \frac{\partial}{\partial x}+\frac{1}{2} y \frac{\partial}{\partial y}, x^{2} \frac{\partial}{\partial x}+x y \frac{\partial}{\partial y}\right\}, \\
\left\{\frac{\partial}{\partial y}, x \frac{\partial}{\partial y}, x^{2} \frac{\partial}{\partial y}, x^{3} \frac{\partial}{\partial y}, y \frac{\partial}{\partial y}, \frac{\partial}{\partial x},\right. \\
\left.x \frac{\partial}{\partial x}+\frac{3}{2} y \frac{\partial}{\partial y}, x^{2} \frac{\partial}{\partial x}+3 x y \frac{\partial}{\partial y}\right\} .
\end{gathered}
$$

The five-dimensional algebra is $A_{2} \oplus_{s} A_{3,3}$. The latter subalgebra is also known as $D \oplus_{s} T_{2}$, i.e., dilations and translations in the plane.

The six-dimensional algebra is $A_{3,3} \oplus_{s} s l(2, R)$. We note that these symmetries are the same as those of $y^{\prime \prime}=0$ apart from the two noncartan symmetries typical of a linear second-order equation [3]. 
The eight-dimensional algebra is the maximal algebra for a forth-order scalar equation. This occurs for $q=-3$. Then $(2)$ is simply $y^{(4)}=0$ up to a multiplier.

Unlike in the case of the third-order equation there is no existence of an $n+3$-dimensional algebra.

This pattern of behaviour persists mutatis mutandis for higher-order equations. The general $n$ th-order equation has three possible numbers of symmetries, namely $n+1, n+2$ and $n+4$. The algebras are as delineated above. We note that the exceptional property of the third-order equation, the possession of a double $\operatorname{sl}(2, R)$ algebras, is indeed exceptional.

\section{Conclusion}

In 12 we considered a family of ordinary differential equations

$$
(n-1) y^{(n-2)} y^{(n)}-n\left(y^{(n-1)}\right)^{2}=0
$$

as a natural generalisation of the well-known KummerSchwarz Equation

$$
2 y^{\prime} y^{\prime \prime \prime}-3\left(y^{\prime \prime}\right)^{2}=0 .
$$

We reported the symmetries, singularity properties and solutions for the members of the family. These properties were remarkably robust throughout the whole family except for the Kummer-Schwarz Equation itself which had the additional property of six Lie Point Symmetries that makes it exceptional in the class of third-order equations.

In this paper we have considered a variation on the Kummer-Schwarz Equation and its natural generalisation to higher-order equations by the inclusion of a parameter $q$ to give the nonlinear family

$$
(n+q-2) y^{(n-2)} y^{(n)}-(n+q-1)\left(y^{(n-1)}\right)^{2}=0 .
$$

There is no a priori constraint upon the value of $q$. Apart from some special values noted in $\S 3$ the value of $q$ does not influence the symmetry properties, i.e., in general the number of symmetries is the same independently of the value of $q$. However, when one considers the singularity analysis, $q$ must necessarily be positive to enable the existence of a singularity, indeed a positive integer for the usual analysis to apply. The value of $q$ affects only the leading-order term. Independently of the value of $q$ the resonances take the values -1 and 0 . Thereafter the value of $q$ enters into the values of the resonances.

\section{ACKNOWLEDGEMENTS}

R Sinuvasan thanks the University Grants Commission for its support. PGLL thanks Professor KM Tamizhmani and the Department of Mathematics, University of Pondicherry, for the provision of facilities whilst this work was undertaken. PGLL also thanks the University of KwaZulu-Natal and the National Research Foundation of the Republic of South Africa for their continued support. Any views expressed in this paper are not necessarily those of the two institutions.

\section{REFERENCES}

[1] Andriopoulos, K., Leach, P. G. L.: An interpretation of the presence of both positive and negative nongeneric resonances in the singularity analysis. Physics Letters A, 359, 2006, p. 199-203. (doi.org/10.1016/j.physleta 2006.06.026)

[2] Feix, M. R., Geronimi, C., Leach, P. G. L., Lemmer, R. L., Bouquet, S.: On the singularity analysis of ordinary differential equations invariant under time translation and rescaling. Journal of Physics A: Mathematical and General, 30(21), 1997, p. 7437-7461. (doi.org/10.1088/0305-4470/30/21/017)

[3] Hsu, L., Kamran, N.: Classification of second-order ordinary differential equations admitting Lie groups of fibre-preserving point symmetries. Proceedings of the London Mathematical Society, 58, 1989, p. 387-416. (doi.org/10.1112/plms/s3-58.2.387)

[4] Kummer, E. E.: De generali quadam æquatione differentiali tertii ordinis. Journal der Reine und Angewandte Mathematik, 100, 1887, p. 1-9. (reprinted from the Programm des evangelischen Königl und Stadtgymnasiums in Liegnitz for the year 1834) (doi.org/10.1515/crll.1887.100.1)

[5] Morozov, V. V., Classification of six-dimensional nilpotent Lie algebras. Izvestia Vysshikh Uchebn Zavendeniĭ Matematika, 5, 1958, p. 161-171.

[6] Mubarakzyanov, G. M.: On solvable Lie algebras. Izvestia Vysshikh Uchebn Zavendeniǔ Matematika, 32, p. 114-123.

[7] Mubarakzyanov, G. M.: Classification of real structures of five-dimensional Lie algebras. Izvestia Vysshikh Uchebn Zavendeniǔ Matematika, 34, 1963, p. 99-106.

[8] Mubarakzyanov, G. M.: Classification of solvable six-dimensional Lie algebras with one nilpotent base element. Izvestia Vysshikh Uchebn Zavendeniu Matematika, 35, 1963, p. 104-116.

[9] Patera, J., Sharp, R. T., Winternitz, P., Zassenhaus, H.: Invariants of real low dimension Lie algebras. Journal of Mathematical Physics, 17(6), 1976, 986-994. (doi.org/10.1063/1.522992)

[10] Ramani, A., Grammaticos, B., Bountis, T.: The Painlevé property and singularity analysis of integrable and nonintegrable systems. Physics Reports, 180, 1989, p. 159-245. (doi.org/10.1016/0370-1573(89)90024-0)

[11] Shabanskaya, A., Thompson, G.: Six-dimensional Lie algebras with a five-dimensional nilradical. Journal of Lie Theory, 23(2), 2013, p. 313-355.

[12] Sinuvasan, R., Tamizhmani, K. M., Leach, P. G. L.: Algebraic and Singularity Properties of a Class of Generalisations of the Kummer-Schwarz Equation. Differential Equations and Dynamical Systems, 2016, doi:10.1007/s12591-016-0327-5.

[13] Ŝnobl, L., Winternitz, P.: Classification and identification of Lie algebras 33. American Mathematical Society. Providence, R.I. 2014.

[14] Tabor, M.: Chaos and Integrability in Nonlinear Dynamics. New York: John Wiley, 1989.

(ISBN:978-0-471-82728-3) 